\section{Síndrome de Doege-Potter: hipoglicemia secundaria a tumor fibroso solitario de la pleura}

\author{
RODRIGO CAMPOS M. ${ }^{1}$, ROBERTO GONZÁLEZ L. ${ }^{1}$, RENÉ SALDÍAS F. ${ }^{1}$, \\ RODRIGO KLAASSEN P. ${ }^{2}$, EMILIO ALARCÓN C. ${ }^{1}$
}

\section{Doege-Potter syndrome: hypoglycemia secondary to solitary fibrous tumor of the pleura. Report of one case}

Doege-Potter syndrome is characterized for hypoglycemia associated with solitary pleural fibrous tumors. We report a 38-year-old woman with a history of weight loss, malaise and edema. After an episode of symptomatic hypoglycemia, she was admitted to the hospital, where she had new episodes of hypoglycemia. A Chest X ray and scan showed a right pleural tumor that was surgically excised. After surgery the episodes of hypoglycemia subsided. The pathological study of the tumor revealed a solitary fibrous pleural tumor. After 15 months of follow up, the patient is symptom free and without evidence of tumor relapse.

(Rev Med Chile 2012; 140: 353-357).

Key words: Hypoglycemia; Pleural neoplasms; Solitary fibrous tumor, pleural.

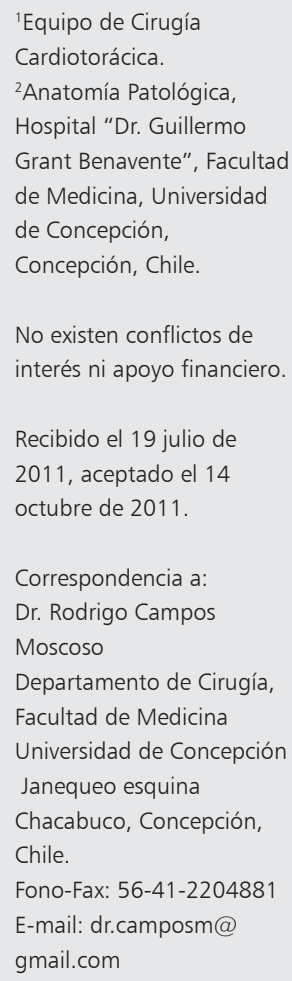

E 1 síndrome de Doege-Potter (SDP) es un síndrome paraneoplásico infrecuente, consiste en la presencia de hipoglicemia sintomática asociada generalmente a un tumor fibroso solitario de la pleura (TFSP), aunque ha sido asociado a otros tumores intratorácicos y a tumores fibrosos en otras localizaciones ${ }^{1-4}$. Fue descrito en forma separada por Karl Doege y Roy Potter en 1930, en un paciente con un tumor fibroso en el mediastino que presentó hipoglicemia ${ }^{5,6}$.

El TFSP es una neoplasia infrecuente, se presenta generalmente en relación a la pleura visceral y representa menos de 5\% de los tumores pleurales. Una manifestación infrecuente en estos pacientes es la hipoglicemia, que se presenta sólo entre 2-3\% de los $\operatorname{casos}^{7,8}$.

Presentamos el caso de una paciente con SDP en que la hipoglicemia sintomática se asoció a un tumor fibroso solitario de la pleura.

\section{Caso clínico}

Mujer de 38 años, domiciliada en sector rural y sin antecedentes mórbidos conocidos. Inició cuadro clínico caracterizado por deterioro del estado general, baja de peso (aproximadamente $10 \mathrm{~kg}$ en tres meses) y edema de extremidades inferiores.

Consultó en varias oportunidades en el centro de salud rural de su sector donde se trató en forma sintomática. Presentó un episodio de hipoglicemia $(26 \mathrm{mg} / \mathrm{dl})$ que se trató con infusión de solución glucosada al 10\%. Evolucionó con normalización de la glicemia y fue trasladada a nuestro hospital.

En la radiografía de tórax se observó una ocupación pleural derecha y desviación de las estructuras mediastínicas (Figura 1A). En la tomografía computada de tórax se observó un tumor pleural derecho, con densidad heterogénea, que ocupaba el hemitórax derecho con efecto compresivo, atelectasia pulmonar derecha y desplazamiento del mediastino (Figura 1B, 1C y 1D). En los otros exámenes de laboratorio y de imagen no destacaron otros hallazgos significativos.

Durante la hospitalización presentó varios episodios de hipoglicemia sintomática caracterizada por somnolencia, deterioro de la agudeza visual, 

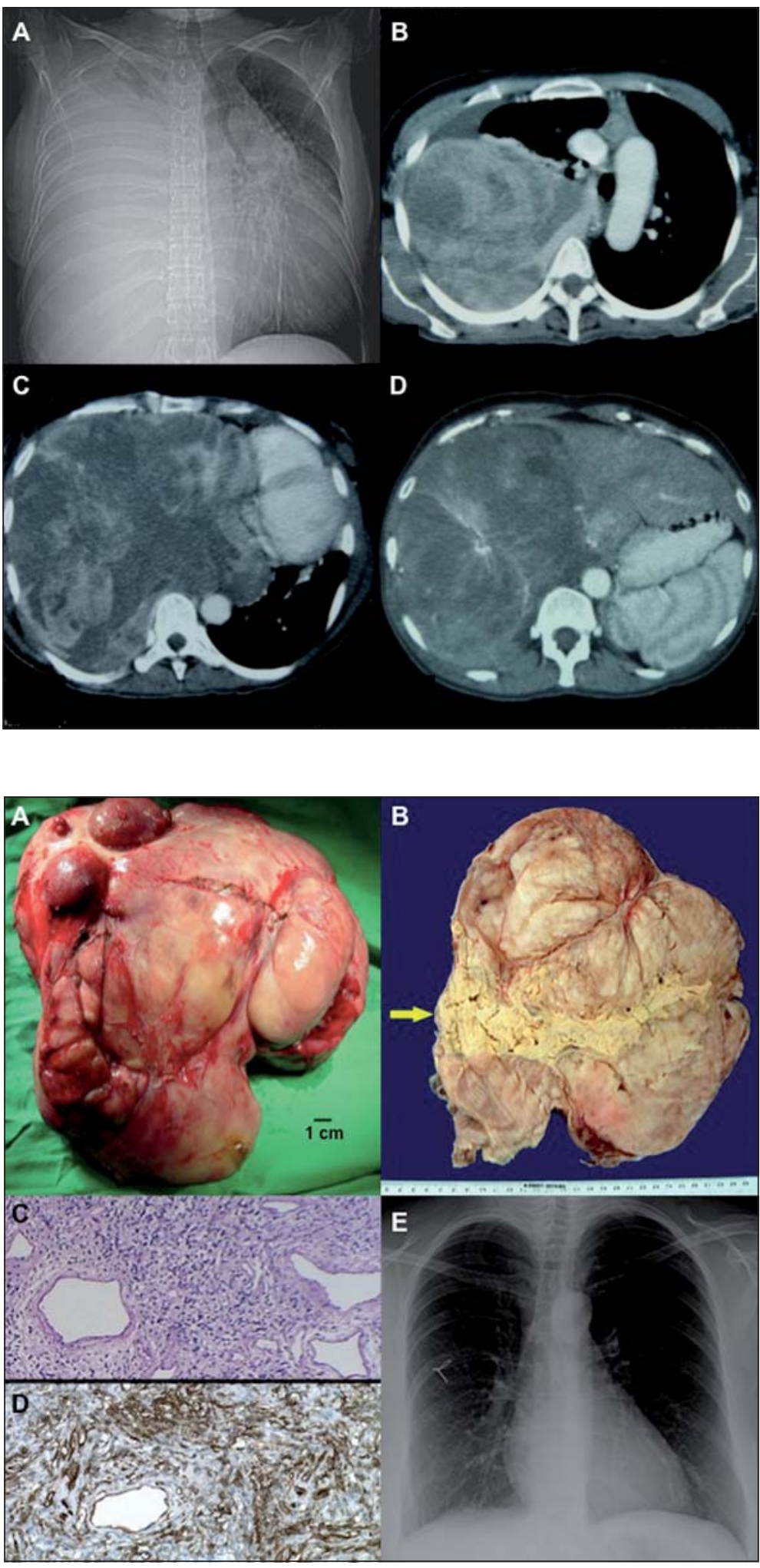

Figura 1. A. Radiografía de tórax postero anterior preoperatoria: se observa ocupación pleural derecha y desviación de estructuras mediastínicas. B, C y D. Tomografía computada de tórax: se observa tumor pleural derecho que ocupa toda cavidad pleural, produce desviación y compresión de corazón y otras estructuras mediastínicas.

Figura 2. A. Foto de tumor resecado (cm: centímetro). B. Foto macroscópica de tumor con necrosis extensa: área amarillenta que cruza horizontalmente la neoplasia (flecha). C. Microscopia: Tumor formado por células fusadas con numerosos vasos sanguíneos. H\&E 100X. D. Inmunohistoquímica con positividad intensa para CD34. E. Radiografía de tórax postero anterior postoperatoria. 


\section{Tabla 1. Episodios de hipoglicemia registrados según: el día de evolución (desde el primer episodio), relación horaria}

\begin{tabular}{|clc|}
\hline Día & Horario / Relación con ingesta & $\begin{array}{c}\text { Glicemia } \\
\text { (mg/dl) }\end{array}$ \\
\hline 1 & PM / Postprandial & 26 \\
\hline 2 & AM / Ayuno & 56 \\
\hline 2 & PM / Preprandial & 43 \\
\hline 4 & AM / Ayuno & 43 \\
\hline 6 & PM / Preprandial & 29 \\
\hline 8 & PM / Preprandial & 41 \\
\hline 11 & AM / Ayuno & 27 \\
\hline 13 & PM / Preprandial & 54 \\
\hline 17 & PM / Postprandial & 58 \\
\hline
\end{tabular}

AM: antes del meridiano / PM: después del meridiano y relación con la ingesta de alimentos (en el segundo día se registraron dos episodios de hipoglicemia).

debilidad muscular, sudoración, palpitaciones y palidez. Estas hipoglicemias fueron en días diferentes y se registraron al menos 7 mediciones de glicemia entre 27 y $58 \mathrm{mg} / \mathrm{dl}$, en el segundo día de hospitalización se registraron dos hipoglicemias (Tabla 1). Fue tratada en cada episodio con infusión de solución glucosada al 10\%. Debido al difícil control metabólico, se trató y estudió en conjunto con el equipo de endocrinología. Las mediciones de insulinemia basal, cortisol, prolactina y hormona tiroestimulante (TSH) estaban en rangos de normales.

Se realizó una biopsia por punción guiada por tomografía computada, el estudio de anatomía patológica concluyó que la lesión era compatible con un TFSP.

Descartada otra causa de hipoglicemia y en el contexto de un TFSP, se decidió tratamiento quirúrgico.

\section{Procedimiento quirúrgico}

Con intubación monobronquial (tubo endotraqueal de doble lumen) y en decúbito lateral izquierdo. Se abordó por amplia toracotomía posterolateral derecha, se encontró un tumor de aspecto encapsulado que ocupaba toda la cavidad pleural y desplazaba las estructuras mediastínicas sin infiltrarlas. Se encontraba adherido a la pleura parietal, con base de implantación ancha y sin pedículo identificable. Se liberaron las adherencias y se resecó macroscópicamente el tumor en forma completa (Figura 2A). Se observó adecuada expansión pulmonar derecha post exéresis del tumor. Se instalaron dos tubos pleurales (28 Fr.) y se cerró la toracotomía en forma habitual.

\section{Evolución postoperatoria}

La paciente evolucionó en el postoperatorio sin incidentes. No presentó nuevos episodios de hipoglicemia. Se retiraron los tubos pleurales al séptimo día y se dio el alta hospitalaria al décimo día postoperatorio.

El estudio anatomopatológico concluyó: TFSP de 27 x 25 x 11,5 centímetros y peso de 3.450 gramos, con características histológicas de malignidad. El TFSP tenía necrosis tumoral macro y microscópica extensa, hasta 2 mitosis en 10 campos de aumento mayor y los bordes quirúrgicos estaban libres de lesión (Figuras 2B y 2C). La inmunohistoquímica presentó positividad intensa para Vimentina y CD34 (Figura 2D).

Actualmente, a 15 meses de seguimiento, la paciente se encuentra con un control metabólico adecuado, con normalización de la glicemia y asintomática desde el punto de vista respiratorio, sin evidencias clínicas ni radiológicas de recidiva del tumor (Figura 2E).

\section{Discusión}

Los TFSP corresponden a tumores primarios de la pleura que se consideran benignos. Son tumores infrecuentes, localizados, de lento crecimiento, poco sintomáticos y pueden alcanzar grandes tamaños. El tratamiento es la resección quirúrgica con buenos resultados inmediatos y escasa recidiva ${ }^{7-10}$.

El tumor fibroso solitario es una neoplasia bien circunscrita, no encapsulada, formada por células fusadas con variabilidad en los patrones de crecimiento de las células fusiformes dentro de una misma lesión. Presenta áreas fibrosas o esclerosadas y un componente vascular prominente. La morfología nuclear es de bajo grado, tiene escasas mitosis, ausencia de necrosis o de pleomorfismo nuclear. Hay características histológicas que se asocian a comportamiento maligno como la presencia de necrosis macro y microscópica y mitosis frecuentes, esto se traduciría en mayor recidiva post resección quirúrgica ${ }^{11}$. 
No sólo ha sido descrito en relación a la pleura, sino que también en órbita, nasofaringe, peritoneo, retroperitoneo, mama, mediastino y otras localizaciones. En el caso de tumores fibrosos solitarios de localización torácica, deben considerarse diagnósticos diferenciales como: sarcoma sinovial, mesotelioma fusocelular y tumor neural como schwannoma. Para esto se utiliza una técnica de diagnóstico complementaria, como la inmunohistoquímica. El tumor fibroso solitario es típicamente positivo para CD34 y Vimentina ${ }^{7,8,11}$.

El TFSP es más frecuentes entre la sexta y séptima década de la vida, sin diferencias significativas en la distribución por género. Al momento del diagnóstico muchos pacientes se encuentran asintomáticos, por lo que éste se realiza en forma incidental. Cuando aparecen síntomas, éstos generalmente son inespecíficos como tos, disnea, dolor torácico y pérdida de peso ${ }^{7,8}$.

La hipoglicemia es una manifestación infrecuente de los TFSP, en una importante serie nacional comunicada, se describe hipoglicemia en un paciente entre 41 casos $^{8}$. La presentación de hipoglicemia sintomática en el contexto de este tipo de neoplasia es lo que conocemos como SDP ${ }^{12,13}$.

El SDP es poco frecuente, en la mayoría de los casos ha sido asociado a tumores fibrosos intratorácicos, aunque también se ha asociado a otro tipo de tumores intratorácicos y a tumores fibrosos extratorácicos ${ }^{1-4,14}$.

Como factores responsables de la hipoglicemia en el SDP se han postulado varias teorías: secreción excesiva por el tumor de IGF-II (Factor de crecimiento similar a la insulina), falla en los mecanismos compensadores para prevenir la hipoglicemia y acelerado consumo de glucosa por tumores de gran tamaño ${ }^{1,2,14-17}$.

La hipoglicemia se ha comunicado en pacientes que presentaron concomitantemente un aumento de IGF-II. En estos casos, tanto los síntomas, como el aumento de IGF-II desparecieron después de la resección del tumor, por lo que se presume que el IGF-II es producido por el tumor pleural ${ }^{15,16}$.

La determinación de IGF-II requiere implementación de laboratorio que no esta disponible en nuestro laboratorio habitual, por este motivo no siempre es medida en los casos de SDP (en nuestro caso no fue determinado).

A modo de conclusiones podemos señalar que: ante la ocurrencia de hipoglicemia sintomática repetida (sin relación a causas como dia- betes mellitus, uso de hipoglicemiantes $\mathrm{u}$ otras endrocrinopatías) debe descartase un síndrome paraneoplásico tipo SDP; que los TFSP pueden manifestarse clínicamente con hipoglicemia sintomática y que el tratamiento quirúrgico esta indicado en estos pacientes.

\section{Referencias}

1. Chamberlain MH, Taggart DP. Solitary fibrous tumor associated with hypoglycemia: an example of the DoegePotter syndrome. J Thorac Cardiovasc Surg 2000; 119: 185-7.

2. Kalebi AY, Hale MJ, Wong ML, Hoffman T, Murray J. Surgically cured hypoglycemia secondary to pleural solitary fibrous tumour: case report and update review on the Doege-Potter syndrome. J Cardiothorac Surg 2009; 4: 45 .

3. Lucas CE, Ledgerwood AM. Malignant solitary fibrous tumor of the intestine with refractory hypoglycemia (Doege Potter Syndrome). J Am Coll Surg 2006; 203: 398.

4. Milenkovic BA, Stojsic, JM, Motohiko A, Dudvarski A, Jakovic R, Stevic R, et al. Solitary fibrous pleural tumor associated with loss of consciousness due to hypoglycemia. Med Oncol 2009; 26: 131-5.

5. Doege KW. Fibro-sarcoma of the mediastinum. Ann Surg 1930; 92: 955-60.

6. Potter RP. Intrathoracic tumors. Radiology 1930; 14: 60-1.

7. Cardillo G, Carbone L, Carleo F, Masala N, Graziano P, Bray A, et al. Solitary fibrous tumors of the pleura: an analysis of 110 patients treated in a single institution. Ann Thorac Surg 2009; 88: 1632-7.

8. Santolaya R, Meneses M, López J, Prats R, Fica M, González C, et al. Tumor fibroso solitario de la pleura. Análisis de 41 casos. Rev Chil Enf Respir 2007; 23: 11-6.

9. Takahama M, Kushibe K, Kawaguchi T, Kimura M, Taniguchi S. Video-assisted thoracoscopic surgery is a promising treatment for solitary fibrous tumor of the pleura. Chest 2004; 125: 1144-7.

10. Sung SH, Chang JW, Kim J, Lee KS, Han J, Park SI. Solitary fibrous tumors of the pleura: surgical outcome and clinical course. Ann Thorac Surg 2005; 79: 303-7.

11. England DM, Hochholzer L, McCarthy MJ. Localized benign and malignant fibrous tumors of the pleura. A clinicopathologic review of 223 cases. Am J Surg Pathol 1989; 13: 640-58.

12. Balduyck B, Lauwers P, Govaert K, Hendriks J, De Maeseneer M, Van Schil P. Solitary fibrous tumor of the pleura with associated hypoglycemia: Doege-Potter 
syndrome: a case report. J Thorac Oncol 2006; 1: 588-90.

13. Zafar H, Takimoto CH, Weiss G. Doege-Potter syndrome: hypoglycemia associated with malignant solitary fibrous tumor. Med Oncol 2003; 20: 403-8.

14. Herrmann BL, Saller B, Kiess W, Morgenroth K, Drochner K, Schröder T, et al. Primary malignant fibrous histiocytoma of the lung: IGF-II producing tumor induces fasting hypoglycemia. Exp Clin Endocrinol Diabetes 2000; 108: 515-8.

15. Fukasawa $\mathrm{Y}$, Takada A, Tateno M, Sato H, Koizumi M,
Tanaka A, et al. Solitary fibrous tumor of the pleura causing recurrent hypoglycemia by secretion of insulinlike growth factor II. Pathol Int 1998; 48: 47-52.

16. Sakamoto T, Kaneshige H, Takeshi A, Tsushima T, Hasegawa S. Localized pleural mesothelioma with elevation of high molecular weight insulin-like growth factor II and hypoglycemia. Chest 1994; 106: 965-7.

17. Fung EC, Crook MA. Doege-Potter syndrome and 'bigIGF2': a rare cause of hypoglycaemia. Ann Clin Biochem 2011; 48: 95-6. 